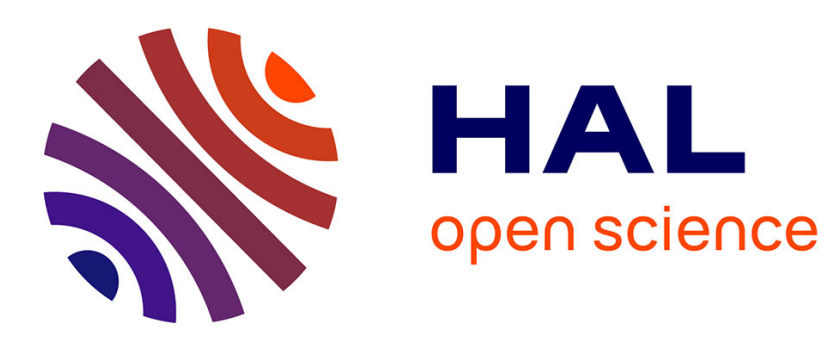

\title{
Etude structurale de $\gamma-\mathrm{Cd}(\mathrm{OH}) 2$ ou $\mathrm{Cd} 2 \mathrm{O}(\mathrm{OH}) 2(\mathrm{H} 2 \mathrm{O})$
}

A Riou, Yannick Cudennec, Y Gerault

\section{To cite this version:}

A Riou, Yannick Cudennec, Y Gerault. Etude structurale de $\gamma-\mathrm{Cd}(\mathrm{OH}) 2$ ou $\mathrm{Cd} 2 \mathrm{O}(\mathrm{OH}) 2(\mathrm{H} 2 \mathrm{O})$. Materials Research Bulletin, 1990, 25 (8), pp.987 - 996. 10.1016/0025-5408(90)90005-m . hal-03271407

\section{HAL Id: hal-03271407 https://hal.science/hal-03271407}

Submitted on 25 Jun 2021

HAL is a multi-disciplinary open access archive for the deposit and dissemination of scientific research documents, whether they are published or not. The documents may come from teaching and research institutions in France or abroad, or from public or private research centers.
L'archive ouverte pluridisciplinaire HAL, est destinée au dépôt et à la diffusion de documents scientifiques de niveau recherche, publiés ou non, émanant des établissements d'enseignement et de recherche français ou étrangers, des laboratoires publics ou privés. 
Mat. Res. Bull., Vol. 25, pp. 987-996, 1990

https://doi.org/10.1016/0025-5408(90)90005-M

ETUDE STRUCTURALE DE $\gamma-\mathrm{Cd}(\mathrm{OH})_{2}$ ou $\quad \mathrm{Cd}_{2} \mathrm{O}(\mathrm{OH})_{2}\left(\mathrm{H}_{2} \mathrm{O}\right)$

A. RIOU, Y.CUDENNEC et Y.GERAULT

Laboratoire de chimie des matériaux inorganiques et de cristallographie

I.N.S.A

20 avenue des buttes de Coësmes - 35043 RENNES cedex (FRANCE)

(Received June 12, 1990; communicated by E.F. Bertaut)

\section{ABSTRACT}

Single crystals of $\gamma-\mathrm{Cd}(\mathrm{OH})_{2}$ were prepared from an intermediate product, $\mathrm{Na}_{2} \mathrm{Cd}(\mathrm{OH})_{4}$. The crystallographic study was conducted on an automatic diffractometer at room temperature and at $133 \mathrm{~K}$. The final $\mathrm{R}$ value for 471 unique reflections was $2.7 \%(133 \mathrm{~K})$. The structural study shows that , $\gamma-\mathrm{Cd}(\mathrm{OH})_{2}$ has a three-dimensional linkage whereas weak hydrogen bonds are involved. The $\mathrm{H}$ atomic positions obtained at $133 \mathrm{~K}$, are incompatible with a common hydroxide because of the presence of a water-like molecule. $\gamma-\mathrm{Cd}(\mathrm{OH})_{2}$ must be considered rather than an aquoxy - hydroxide of formulae: $\mathrm{Cd}_{2} \mathrm{O}(\mathrm{OH})_{2}\left(\mathrm{H}_{2} \mathrm{O}\right)$. All these results are confirmed by the I.R spectrum.

The cell parameters at $133 \mathrm{~K}$ are:

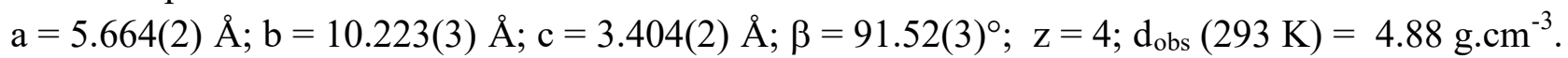
The space group is Im.

. MATERIALS INDEX: cadmium, hydroxides

\section{INTRODUCTION}

L'hydroxyde de cadmium $\mathrm{Cd}(\mathrm{OH})_{2}$ joue un rôle important dans le fonctionnement de l'accumulateur au cadmium-nickel. Il constitue en effet la matière active anodique de l'accumulateur à l'état déchargé. C'est pourquoi de nombreux travaux lui ont été consacrés. Ces travaux sont rappelés dans la revue bibliographique de BARNARD (1).

Dans une étude physico-chimique récente (2),(3), nous avons élucidé le mécanisme de la formation de $\gamma-\mathrm{Cd}(\mathrm{OH})_{2}$ et effectué l'étude structurale à partir du diagramme de poudre. Cette étude structurale a permis de mieux préciser les résultats antérieurement publiés par DE WOLFF (4) qui avait étudié un hydroxyfluorure de cadmium. 
Très récemment nous avons mis au point un procédé permettant de fabriquer des monocristaux de $\gamma-\mathrm{Cd}(\mathrm{OH})_{2}$, ce qui a permis d'envisager une étude structurale plus approfondie qui fait l'objet de la présente publication.

\section{CRISTALLOGENESE}

Dans notre récente publication (3), nous avons exposé une nouvelle méthode de préparation de l'hydroxyde de cadmium $\gamma-\mathrm{Cd}(\mathrm{OH})_{2}$, par hydrolyse de l'hydroxycadmiate de sodium $\mathrm{Na}_{2} \mathrm{Cd}(\mathrm{OH})_{4}$.

Les systèmes de départ sont constitués par un mélange homogène de soude et d'oxyde de cadmium $\mathrm{CdO}$. Ils sont hydratés très lentement, à l'abri du dioxyde de carbone de l'air, dans une enceinte étanche dans laquelle on a placé un dispositif d'émission contrôlée de vapeur d'eau. On met alors à profit les propriétés d'hygroscopicité de la soude pour hydrater lentement le mélange réactionnel de départ. Il se forme alors l'hydroxycadmiate de sodium, selon la réaction suivante:

$$
2 \mathrm{NaOH}+\mathrm{CdO} \underset{\text { Tambiante }}{\mathrm{H}_{2} \mathrm{O}(\mathrm{gaz})} \triangleright \mathrm{Na}_{2} \mathrm{Cd}(\mathrm{OH})_{4}
$$

La présence d'un excès d'eau et de soude par rapport aux proportions stoechiométriques, est nécessaire pour augmenter la vitesse de réaction entre deux phases solides à température ambiante.

L'étude d'un système constitué de 6.4 moles de soude pour 1 mole de $\mathrm{CdO}$, s'est révélée fructueuse pour l'obtention de monocristaux de taille suffisante pour une étude cristallographique complète. L'évolution du système est suivie par pesées régulières effectuées à l'abri de l'air, afin de pouvoir situer le point figuratif de celui-ci dans le diagramme ternaire $\mathrm{CdO}, \mathrm{Na}_{2} \mathrm{O}, \mathrm{H}_{2} \mathrm{O}(3)$. Après une semaine de réaction, la formation de l'hydroxy-cadmiate de sodium est pratiquement terminée. Le système a l'aspect d'une pâte gélatineuse blanche de très faible granulométrie.

L'hydrolyse est alors poursuivie avec une extrême lenteur pendant environ trois mois et la réaction donne progressivement naissance à l'hydroxyde de cadmium, selon le schéma réactionnel suivant :

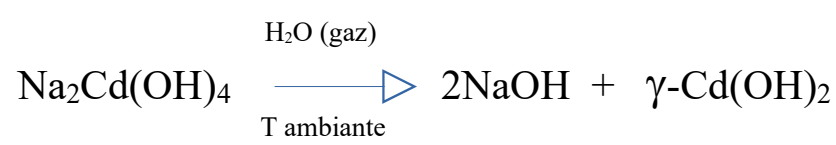

Les cristaux de $\quad \gamma-\mathrm{Cd}(\mathrm{OH})_{2} \quad$ apparaissent sous la forme d'aiguilles transparentes aplaties dont la longueur moyenne est voisine de $4 / 10 \mathrm{de} \mathrm{mm}$.

\section{ETUDE CRISTALLOGRAPHIQUE}

Une étude préliminaire du cristal sur chambres photographiques, confirme les valeurs des paramètres de la maille et du groupe spatial $(\mathrm{Im})$ initialement déterminés à partir d'une poudre. Les paramètres de la. maille ont été affinés par moindres-carrés à partir de 25 réflexions correspondant à des valeurs de $\theta$ comprises entre 5 et $25^{\circ}$ et optimisés sur diffractomètre automatique. La masse volumique expérimentale a été mesurée par la méthode de la poussée d'Archimède, dans le bromobenzène. Un premier enregistrement des intensités diffractées a été effectué à température ambiante, sur diffractomètre automatique ENRAF-NONIUS. Le monochromateur utilisé était à lame de graphite et la distance cristal-détecteur était de $173 \mathrm{~mm}$. L'enregistrement a été réalisé pour des valeurs de $\theta$ comprises entre 1 et $32^{\circ}$, avec un balayage $\omega-2 \theta$, une amplitude de balayage: $(1.00+0.35 \tan \theta)^{\circ}$ et une ouverture de $(2.00+0.50 \tan \theta)^{\circ} .519$ réflexions indépendantes ont été mesurées et corrigées du facteur de LORENTZ-polarisation. Les dimensions du cristal étudié 
étaient de: 300 × $100 \times 6(\mu \mathrm{m})$. Des corrections d'absorption ont été effectuées par la méthode de WALKER and STUART (5). Au cours de l'enregistrement les 3 réflexions standards n'ont pas subi de variation significative. 506 réflexions avec I $>0.5 \sigma(\mathrm{I})$ ont été conservées pour la résolution de la structure. Les calculs ont été réalisés sur P.D.P. 11/60 avec la bibliothèque de programmes cristallographiques S.D.P. (6). Les facteurs de diffusion utilisés sont de CROMER et WABER (7). Tous les affinements de structure ont été réalisés par une méthode de moindre-carrés à matrice totale en minimisant la fonction $\Sigma \mathrm{w}(\Delta \mathrm{F})^{2}$. Les positions initiales des atomes de cadmium et d'oxygène étaient celles déduites de 1'étude sur poudre. Une première série d'affinements effectuée en considérant les facteurs d'agitation thermique isotrope, conduit à un indice résiduel $\mathrm{R}$ de $5 \%$. Une seconde série réalisée avec des coefficients d'agitation thermique anisotrope, conduit à une valeur de $3.8 \%$. A ce stade de la détermination structurale, la recherche des positions des atomes d'hydrogène par Fourier-différence n'a pas permis de déterminer les positions de tous les atomes d'hydrogène.

Un deuxième enregistrement a alors été réalisé dans les mêmes conditions que le précédent mais à basse température $(133 \mathrm{~K})$. Ce deuxième enregistrement a été effectué à partir du même cristal et a permis de mesurer 471 réflexions indépendantes [I $>1.0 \sigma(\mathrm{I})$ ]. L'affinement des positions et des coefficients d'agitation thermique anisotrope des atomes d'oxygène et de cadmium effectué a partir de cet enregistrement, fournit des résultats très proches de ceux obtenus à température ambiante; les coefficients d'agitation thermique ayant toutefois des valeurs plus faibles. L'analyse des séries de Fourier-différence calculées après cet affinement, montre que trois seulement des pics observés peuvent être imputés à des atomes d'hydrogène. Une nouvelle série d'affinements effectuée à partir de toutes les positions atomiques mais en fixant les positions des hydrogènes, conduit à une valeur résiduel $\mathrm{R}$ de $2.7 \%$ et du facteur pondéré $\mathrm{Rw}$ de $3.1 \%$. Les valeurs des paramètres de position et des coefficients d'agitation thermique obtenues à température ambiante et à $133 \mathrm{~K}$, sont rassemblées dans le tableau $\mathrm{I}$.

\section{RESULTATS ET DISCUSSION}

La structure de $\gamma-\mathrm{Cd}(\mathrm{OH})_{2}$ (FIG.1) contient un cadmium et trois oxygènes indépendants. Les valeurs des distances $\mathrm{Cd}-\mathrm{O}$ et des angles $\mathrm{O}-\mathrm{Cd}-\mathrm{O}$ sont indiquées dans le tableau II.

Le cadmium est entouré par un octaèdre d'oxygènes très distordu puisque la longueur de la liaison Cd-O est comprise entre 2.25 et $2.44 \AA$ et que l'angle O-Cd-O s'écarte de $15.5^{\circ}$ de l'angle théorique. Les valeurs des distances et des angles ci-dessus ont été calculées à partir de l'étude sur cristal à $133 \mathrm{~K}$. Elles sont assez proches de celles que nous avions obtenues grâce à l'étude sur poudre. Les octaèdres [CdO6] sont associés par paires car ils mettent en commun une face triangulaire constituée par les oxygènes $\mathrm{O}(1), \mathrm{O}^{\prime}(1)$ et $\mathrm{O}(2)$. Ces paires d'octaèdres sont reliées à d'autres paires d'octaèdres identiques par l'intermédiaire d'arêtes d'oxygènes $O(1)--O(3)$. Cet ensemble constitue une double chaîne d'octaèdres qui se développe parallèlement à l'axe « $c$ ». La cohésion latérale entre ces chaînes suivant les deux autres directions de l'espace est assurée par l'oxygène $\mathrm{O}(3)$. Contrairement à la plupart des autres hydroxydes, la structure de $\gamma-\mathrm{Cd}(\mathrm{OH})_{2}$ possède donc un enchaînement tridimensionnel.

Comme nous l'avions déjà montré dans une précédente publication [2], la structure de $\gamma$ $\mathrm{Cd}(\mathrm{OH})_{2}$ possède une autre propriété remarquable: les environnements en cadmium des trois oxygènes sont très différents puisqu'ils sont liés respectivement à 4,2 et 3 atomes de cadmium. Cette propriété peut correspondre au fait que tous les oxygènes de cette structure n'appartiennent pas nécessairement à de véritables groupements $\mathrm{O}-\mathrm{H}$. Nous avions alors émis l'hypothèse que la formule de $\gamma-\mathrm{Cd}(\mathrm{OH})_{2}$ pouvait s'écrire: $\mathrm{Cd}_{2} \mathrm{O}(\mathrm{OH})_{2}\left(\mathrm{H}_{2} \mathrm{O}\right)$. Selon cette hypothèse:

- $\mathrm{O}(1)$ lié à $4 \mathrm{Cd}$ serait un oxygène oxyde

- $\mathrm{O}(2)$ lié à $2 \mathrm{Cd}$ serait un oxygène de molécule d'eau

- $\mathrm{O}(3)$ lié à $3 \mathrm{Cd}$ serait un oxygène $\mathrm{O}-\mathrm{H}$ 
Bien que la recherche des positions des hydrogènes dans un composé aussi riche en élément lourd ait été difficile, les résultats obtenus à partir de l'étude du cristal à $133 \mathrm{~K}$ semble bien confirmer cette hypothèse. En particulier, l'étude des séries de Fourier-différence n'a jamais révélé de pic correspondant à un éventuel atome d'hydrogène relié à l'oxygène $O(1)$. Les valeurs des distances $\mathrm{O}-\mathrm{H}$ et des angles autour de l'oxygène, calculées d'après les positions de tous les atomes sont indiquées dans le tableau III.

L'oxygène $O(1)$ se trouve au sommet d'une pyramide dont la base est formée par 4 cadmiums. La distance séparant $\mathrm{O}(1)$ du plan moyen des $4 \mathrm{Cd}$ est de $0.65 \AA$. L'oxygène $\mathrm{O}(2)$ forme avec les hydrogènes $\mathrm{H}^{\prime}(\mathrm{O} 2)$ et $\mathrm{H}^{\prime}(\mathrm{O} 2)$ une molécule d'eau dont les distances $\mathrm{O}-\mathrm{H}(1.11$ et $1.06 \AA)$ et angle $\mathrm{H}-\mathrm{O}-\mathrm{H}\left(101.8^{\circ}\right)$ sont conformes aux valeurs habituelles. L'environnement complet de $\mathrm{O}(2)$ est un tétraèdre dont les sommets sont occupés par $2 \mathrm{Cd}$ et $2 \mathrm{H}$. L'angle moyen des liaisons est de $106.8^{\circ}$.

L'analyse des positions relatives des atomes d'oxygène montre qu'il n'existe qu'une seule possibilité de formation de liaison par pont hydrogène. Celle-ci s'établit entre les oxygènes $\mathrm{O}(3)$ et $\mathrm{O}(2)$ qui sont distants de $2.86 \AA$ (tableau III). Cette liaison par pont est relativement faible comparée à celle d'autres structures où la distance $\mathrm{O}(\mathrm{H})---\mathrm{O}$ s'abaisse parfois à $2.5 \AA$. Les atomes d'hydrogène $H^{\prime}(\mathrm{O} 2)$ et $H^{\prime}$ ' $(\mathrm{O} 2)$ ne peuvent pas contribuer à la formation de liaisons par pont hydrogène car les oxygènes environnants, seuls susceptibles de pouvoir jouer le rôle d'accepteur sont trop éloignés de $\mathrm{O}(2)$. Il s'agit en fait de $\mathrm{O}(1)$ et d'un atome équivalent de $\mathrm{O}(2)$ qui sont distants respectivement de 3.55 et $3.40 \AA$.

Le spectre infrarouge (I.R.) de $\gamma-\mathrm{Cd}(\mathrm{OH})_{2} \quad$ (Fig.2) fait apparaitre 3 bandes d'absorption caractéristiques des vibrations d'élongation $v(\mathrm{OH})$ à 3580,3530 et $3240 \mathrm{~cm}^{-1}$. Les 2 premières bandes ( 3580 et $3530 \mathrm{~cm}^{-1}$ ) ne peuvent être attribuées qu'à des liaisons O-H peu affectées par des liaisons par pont hydrogène, car elles sont faiblement décalées par rapport aux bandes d'absorption des hydroxyles O-H "libres". Ces bandes pourraient bien correspondre à $\mathrm{O}(3)-\mathrm{H}(\mathrm{O} 3)$ qui forme avec $\mathrm{O}(2)$ une liaison hydrogène faible. En revanche, la bande d'absorption à $3240 \mathrm{~cm}^{-1}$ peut à priori être attribuée, soit à un $\mathrm{OH}$ impliquant une liaison par pont hydrogène de force exceptionnelle , soit à une molécule d'eau. Comme nous venons de le voir précédemment, l'analyse des liaisons hydrogènes possibles au plan structural, montre que, compte tenu des distances inter-oxygène, la structure de $\gamma-\mathrm{Cd}(\mathrm{OH})_{2}$ ne peut renfermer que des liaisons par pont hydrogène faibles. On ne peut donc pas attribuer la bande d'absorption I.R. de $3240 \mathrm{~cm}^{-1}$ à un simple hydroxyle O-H. On peut rappeler à ce sujet un exemple significatif : la structure de Zn3(HPO4)3, 3H2O (8) contient des liaisons hydrogène exceptionnellement fortes puisque la distance inter-oxygène $\mathrm{O}(\mathrm{H})$----O s'abaisse à $2.54 \AA$. Malgré cela, le spectre I.R. de ce composé montre que la bande d'absorption $v(\mathrm{OH})$ la plus décalée ne se situe qu'à $3380 \mathrm{~cm}^{-1}$. Le décalage est donc moins important que celui de la bande à $3240 \mathrm{~cm}^{-1}$ de $\gamma-\mathrm{Cd}(\mathrm{OH})_{2}$.

L'apparition de cette bande particulière dans le spectre I.R. est donc un argument supplémentaire prouvant l'existence d'une molécule d'eau dans $\gamma-\mathrm{Cd}(\mathrm{OH})_{2}$. Il faut toutefois remarquer que cette bande ne possède pas le profil habituel des bandes $v(\mathrm{OH})$ que l'on attribue généralement à la molécule d'eau : elle est relativement fine et bien définie, alors qu'habituellement elles sont plutôt larges et mal définies. D'autre part on n'observe pas dans le spectre I.R., la bande de déformation $\delta(\mathrm{H}-\mathrm{O}-\mathrm{H})$ normalement située vers $1600 \mathrm{~cm}^{-1}$.

\section{CONCLUSION}

Tous les faits qui viennent d'être cités obligent à considérer que l'eau est présente dans la structure de $\gamma-\mathrm{Cd}(\mathrm{OH})_{2}$ sous la forme d'une pseudo-molécule d'eau $\mathrm{H}-\mathrm{O}-\mathrm{H}$ ou eau de constitution (9). Ceci est parfaitement compatible avec les résultats structuraux obtenus par diffraction $\mathrm{X}$ 
puisque les 2 hydrogènes $\mathrm{H}^{\prime}(\mathrm{O} 2)$ et $\mathrm{H}^{\prime}(\mathrm{O} 2)$ sont indépendants ; les distances $\mathrm{O}-\mathrm{H}$ ne sont donc pas égales. Il en résulte par conséquent que les 2 liaisons $\mathrm{O}-\mathrm{H}$ n'ont pas la même force.

Etant donnée la nature particulière de cette pseudo-molécule d'eau, on ne peut pas exclure la possibilité que l'un des hydrogènes, probablement $\mathrm{H}^{\prime}(\mathrm{O} 2)$, car c'est le plus proche de $\mathrm{O}(1)$, puisse se partager entre 2 positions proches de $\mathrm{O}(2)$ et $\mathrm{O}(1)$ suivant le schéma indiqué ci-dessous :

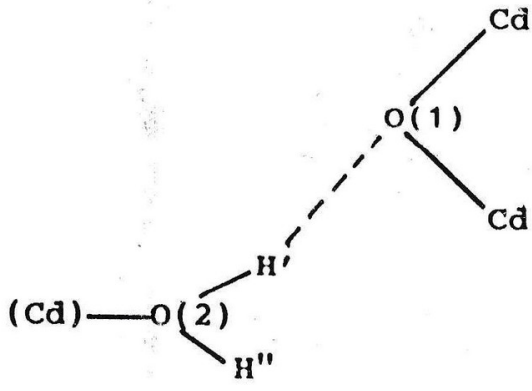

configuration $[A]$

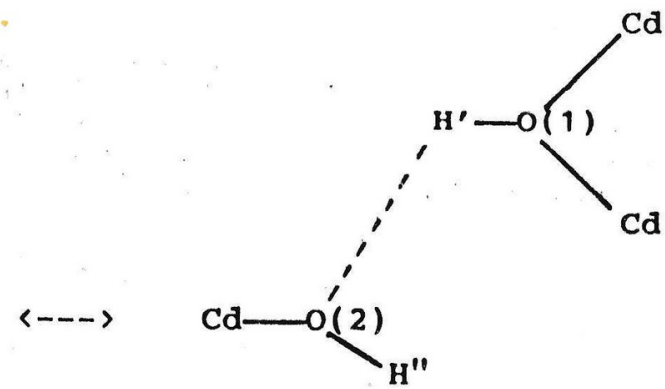

configuration [B]

$\gamma-\mathrm{Cd}(\mathrm{OH})_{2}$ posséderait alors une structure résonnante dont les configurations [A] et [B] seraient les formes limites. Dans la configuration [A] le composé est un aquoxy-hydroxyde de formule $\mathrm{Cd}_{2} \mathrm{O}(\mathrm{OH})_{2}\left(\mathrm{H}_{2} \mathrm{O}\right)$, dans la configuration [B] c'est un simple hydroxyde. Ces deux configurations correspondent en réalité à un désordre de position de l'hydrogène $\mathrm{H}^{\prime}(\mathrm{O} 2)$. Un tel désordre de position de l'hydrogène impliquant son appartenance soit à un hydroxyle $\mathrm{O}-\mathrm{H}$, soit à une molécule d'eau, a déjà été signalé dans le cas des hydroxydes hydratés $\mathrm{Rb}$ et $\mathrm{Cs}(\mathrm{OH}), \mathrm{H}_{2} \mathrm{O}(10)$. L'étude du cristal à $133 \mathrm{~K}$ montre toutefois une prédominance de la configuration [A].

D'après tout ce qui précède, il est clair que $\gamma-\mathrm{Cd}(\mathrm{OH})_{2}$ ne peut pas correspondre à un véritable hydroxyde mais plutôt à un aquoxy-hydroxyde.

Comme nous l'avions déjà montré dans une précédente publication, ce composé est un intermédiaire entre l'oxyde $\mathrm{CdO}$ et l'hydroxyde de cadmium dont la forme stable $\beta-\mathrm{Cd}(\mathrm{OH})_{2}$ possède la structure brucite qui correspond à un véritable hydroxyde (Fig.2).

En outre, une comparaison intéressante peut être établie entre $\gamma-\mathrm{Cd}(\mathrm{OH})_{2}$ et l'hydroxyde de cuivre $\mathrm{Cu}(\mathrm{OH})_{2}$ dont la structure a été déterminée à partir d'une poudre (11). L'étude structurale de ce composé montre que les distances inter-oxygène sont incompatibles avec des liaisons hydrogène fortes. Le spectre I.R. de $\mathrm{Cu}(\mathrm{OH})_{2}$ (Fig.2) montre pourtant une bande d'absorption $\mathrm{v}(\mathrm{OH})$ intense et bien définie à $3300 \mathrm{~cm}^{-1}$. Il paraîtrait dans ce cas, normal d'imputer cette bande à la présence d'une pseudo-molécule d'eau comme pour $\gamma-\mathrm{Cd}(\mathrm{OH})_{2}$. Selon cette hypothèse, $\mathrm{Cu}(\mathrm{OH})_{2}$ qui ne possède pas la structure brucite, pourrait être aussi considéré comme un aquoxy-hydroxyde comparable à $\gamma-\mathrm{Cd}(\mathrm{OH})_{2}$. Cette hypothèse ne pourra être confirmée que grâce à une étude structurale effectuée sur monocristal. Actuellement, des préparations visant à faire croître des monocristaux sont en cours.

Enfin, comme nous l'avons déjà signalé dans de précédentes publications (3), (12), les deux composés $\gamma-\mathrm{Cd}(\mathrm{OH})_{2}$ et $\mathrm{Cu}(\mathrm{OH})_{2}$ peuvent être préparés simplement par hydrolyse des hydroxymétallates $\mathrm{Na}_{2} \mathrm{Cd}(\mathrm{OH})_{4}$ et $\mathrm{Na}_{2} \mathrm{Cu}(\mathrm{OH})_{4}$. Cette analogie dans la préparation n'est peut-être pas sans rapport avec les propriétés de ces composés au niveau structural. 
1. R.Barnard, Journal of Applied Electrochemistry, 11, 217, (1981).

2. A.Lecerf, A.Rlou, Y.Cudennec, Y.Gérault et C.Chanson, Mater. Res. Bul1., 23, 1479-1490, (1988)

3. Y.Cudennec, A.Lecerf, A.Riou et Y.Gérault, Mater. Res. Bull. 24, 381-388, (1989).

4. P.M.De Wolff, Acta Cryst.,21, 432-433, (1966).

5. N.Walker et D.Stuart, Acta Cryst., A39, 159-168, (1983).

6. B.A.Frentz, The Enraf-Nonius CAD-4 S.D.P. system, Delft Untv. Press, (1978).

7. D.T.Cromer et J.T.Waber, Acta Cryst., 18, 104-109, (1965). 8. A.Riou, Y.Cudennec et Y.Gérault, Acta Cryst., C43, 194-197,
(1987).

9. H.D.Lutz, Bonding and structure of Water Molecules in Solid Hydrates, Springer-Verlag Berlin Heidelberg, (1988). 10. H.D.Lutz, J.Henning, H.Jacobs et B.Harbrecht, Ber.

Bunsenges. Phys. Chem., 92, 1557-1561, (1988).

11. H.Jaggi et H.R. Oswald, Acta Cryst., 4, 1041, (1961).

12. Y.Cudennec, A.Lecerf, A.Riou et Y.Gérault, Eur. J. Sol1d

state Inorg. Chem., 25, 351-358, (1988).

TABLEAU I : Positions atomiques et coefficients d'agitation thermique à température ambiante et à $133 \mathrm{~K}$

\begin{tabular}{|lllll|}
\hline & \multicolumn{1}{c}{$x$} & \multicolumn{1}{c}{$y$} & $z$ & \multicolumn{1}{c}{ Beq } \\
Cd & 0,000 & $0,15252(4)$ & 0,000 & $0,871(7)$ \\
& 0,000 & $0,15254(4)$ & 0,000 & $0,446(5)$ \\
O(1) & $0,116(2)$ & 0,000 & $0,489(3)$ & $0,9(1)$ \\
& $0,115(2)$ & 0,000 & $0,489(3)$ & $0,5(1)$ \\
O(2) & $0,689(2)$ & 0,000 & $0,942(4)$ & $1,2(1)$ \\
& $0,687(2)$ & 0,000 & $0,942(3)$ & $0,7(1)$ \\
O(3) & $0,883(1)$ & $0,2790(6)$ & $0,495(2)$ & $0,98(9)$ \\
H' (02) & $0,879(1)$ & $0,2796(6)$ & $0,495(2)$ & $0,54(8)$ \\
H" (02) & 0,000 & 0,500 & 0,332 & 2,0 \\
H (03) & 0,000 & 0,000 & 0,250 & 2,0 \\
\hline
\end{tabular}

\begin{tabular}{|ccccccc|}
\multicolumn{1}{c}{$\beta 11$} & $\beta 22$ & $\beta 33$ & $\beta 12$ & $\beta 13$ & $\beta 23$ \\
\hline \multirow{2}{*}{$C d$} & $0,0069(1)$ & $0,00216(3)$ & $0,0175(3)$ & $-0,0003(2)$ & $-0,0009(3)$ & $0,0004(4)$ \\
& $0,0035(1)$ & $0,00107(2)$ & $0,0096(2)$ & $0,0000(3)$ & $0,0002(2)$ & $-0,0001(6)$ \\
$0(1)$ & $0,007(2)$ & $0,0023(6)$ & $0,020(6)$ & 0 & $0,003(6)$ & 0 \\
& $0,005(2)$ & $0,0010(5)$ & $0,013(5)$ & 0 & $0,003(6)$ & 0 \\
$0(2)$ & $0,007(2)$ & $0,0025(6)$ & $0,033(8)$ & 0 & $-0,006(7)$ & 0 \\
& $0,003(2)$ & $0,0018(6)$ & $0,021(7)$ & 0 & $0,003(6)$ & 0 \\
$0(3)$ & $0,007(1)$ & $0,0031(5)$ & $0,017(4)$ & $0,001(1)$ & $0,001(4)$ & $0,003(3)$ \\
& $0,002(1)$ & $0,0026(4)$ & $0,005(3)$ & $0,000(1)$ & $0,002(4)$ & $0,000(2)$ \\
\hline
\end{tabular}


TABLEAU II : Distances et angles autour du cadmium

\begin{tabular}{|c|c|c|c|c|c|c|}
\hline $\mathrm{Cd}-\mathrm{O}(1)$ & $:$ & $2,438(7)$ & $\AA$ & $O(1)-C d-O^{\prime}(1)$ & $:$ & $90,4(2)^{\circ}$ \\
\hline $\mathrm{Cd}-\mathrm{O}^{\prime}(1)$ & $:$ & $2,361(7)$ & $\AA$ & $o(1)-c d-o(2)$ & $:$ & $74,5(3)^{\circ}$ \\
\hline$C d-O(2)$ & $:$ & $2,367(7)$ & $\AA$ & $O(1)-C a-O(3)$ & : & $84,6(2)^{\circ}$ \\
\hline$C d-O(3)$ & : & $2,248(6)$ & $\AA$ & $O(1)-C d-O^{\prime}(3)$ & : & $175,4(2)^{\circ}$ \\
\hline $\mathrm{Cd}-\mathrm{O}^{\prime}(3)$ & : & $2,249(6)$ & $\AA$ & $O(1)-\mathrm{Cd}-\mathrm{O}^{\prime \prime}(3)$ & : & $85,3(3)^{\circ}$ \\
\hline $\mathrm{Cd}-\mathrm{O}^{\prime \prime}(3)$ & : & $2,255(6)$ & $\AA$ & $O^{\prime}(1)-C d-O(2)$ & $:$ & $79,4(3)^{\circ}$ \\
\hline \multirow[t]{9}{*}{ moyenne } & : & $2,320 \AA$ & & $O^{\prime}(1)-C d-O(3)$ & : & $174,0(2)^{\circ}$ \\
\hline & & & & $0^{\prime}\left(1 ;-\mathrm{Cd}-\mathrm{O}^{\prime}(3)\right.$ & $:$ & $86,4(2)^{\circ}$ \\
\hline & & & & $O^{\prime}(1)-C d-O^{\prime \prime}(3)$ & : & $87,9(3)^{\circ}$ \\
\hline & & & & $O(2)-C d-O(3)$ & : & $95,9(3)^{\circ}$ \\
\hline & & & & $O(2)-C d-O^{\prime}(3)$ & : & $101,5(3)^{\circ}$ \\
\hline & & & & $O(2)-C d-O^{\prime \prime}(3)$ & : & $156,0(2)^{\circ}$ \\
\hline & & & & $O(3)-\mathrm{Cd}-\mathrm{O}^{\prime}(3)$ & $:$ & $98,4(2)^{\circ}$ \\
\hline & & & & $\mathrm{O}(3)-\mathrm{Cd}-\mathrm{O}^{\prime \prime}(3)$ & : & $95,0(2)^{\circ}$ \\
\hline & & & & $O^{\prime}(3)-C d-O^{\prime \prime}(3)$ & $:$ & $97,9(2)^{\circ}$ \\
\hline
\end{tabular}

TABLEAU III : Environnement de 1 'oxygène dans $\gamma-\mathrm{Cd}(\mathrm{OH})_{2}$

\begin{tabular}{|c|c|c|c|c|c|c|}
\hline & $O(1)-C d$ & $: 2,438$ & $(2 x)$ & $C d-O(1)-C d$ & : & 90,4 \\
\hline & $O(1)-C d$ & $: 2,361$ & & $C d-O(1)-C d$ & : & 82,7 \\
\hline & & · & $(2 x)$ & $C d-O(1)-C d$ & : & 148,4 \\
\hline & & & & $C d-O(1)-C d$ & $:$ & 79,5 \\
\hline & 1 & & & & & \\
\hline$(2 x)$ & $O(2)-C d$ & $: 2,367$ & & $\mathrm{Cd}-\mathrm{O}(2)-\mathrm{Cd}$ & : & 82,4 \\
\hline & $(2)-H^{\prime}(02)$ & $: 1,11$ & $(2 x)$ & $\mathrm{Cd}-\mathrm{O}(2)-\mathrm{H}^{\prime}(\mathrm{O} 2)$ & : & 137,1 \\
\hline & (2)-H" (O2) & $: 1,06$ & $(2 x)$ & $\mathrm{Cd}-\mathrm{O}(2)-\mathrm{H}^{\prime \prime}(\mathrm{O} 2)$ & $:$ & 91,1 \\
\hline & & & $\mathrm{H}^{\prime}(\mathrm{O}$ & $2)-O(2)-H^{\prime \prime}(02)$ & : & 101,8 \\
\hline & $O(3)-C d$ & $: 2,249$ & & $\mathrm{Cd}-\mathrm{O}(3)-\mathrm{Cd}$ & $:$ & 98,4 \\
\hline & $O(3)-C d$ & $: 2,248$ & & $C d-O(3)-C d$ & : & 119,5 \\
\hline & $O(3)-C d$ & $: 2,255$ & & $C d-O(3)-C d$ & $:$ & 116,3 \\
\hline & $\mathrm{O}(3)-\mathrm{H}(03)$ & $: 0,87$ & & $\mathrm{Cd}-\mathrm{O}(3)-\mathrm{H}(03)$ & : & 96,6 \\
\hline & & & & $\mathrm{Cd}-\mathrm{O}(3)-\mathrm{H}(03)$ & : & 96,6 \\
\hline & & & & $\mathrm{Cd}-\mathrm{O}(3)-\mathrm{H}(03)$ & : & 124,1 \\
\hline & $(3)---0(2)$ & $: 2,86$ & & (3) $-\mathrm{H}(03)--\mathrm{O}(2)$ & : & 158,6 \\
\hline
\end{tabular}




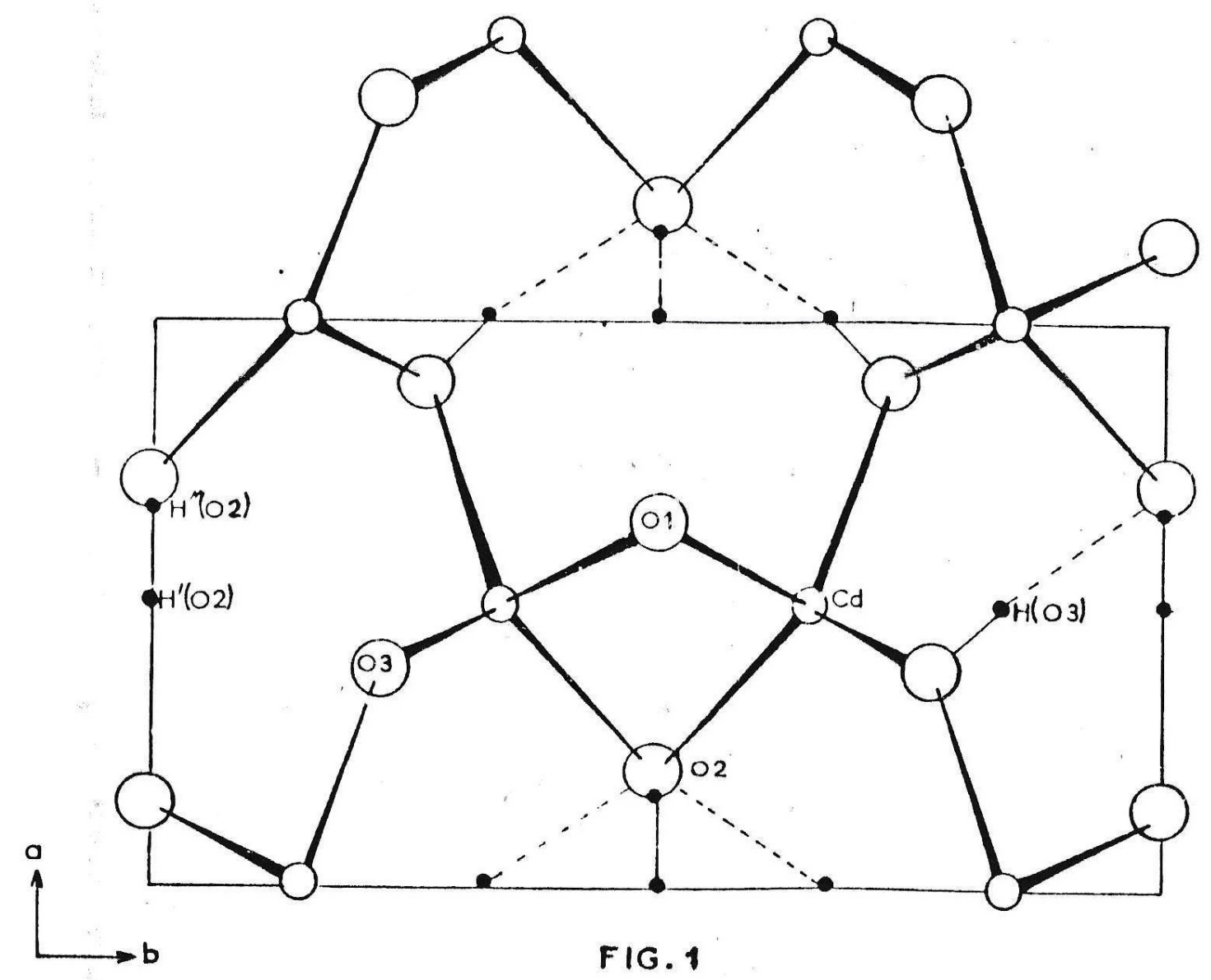




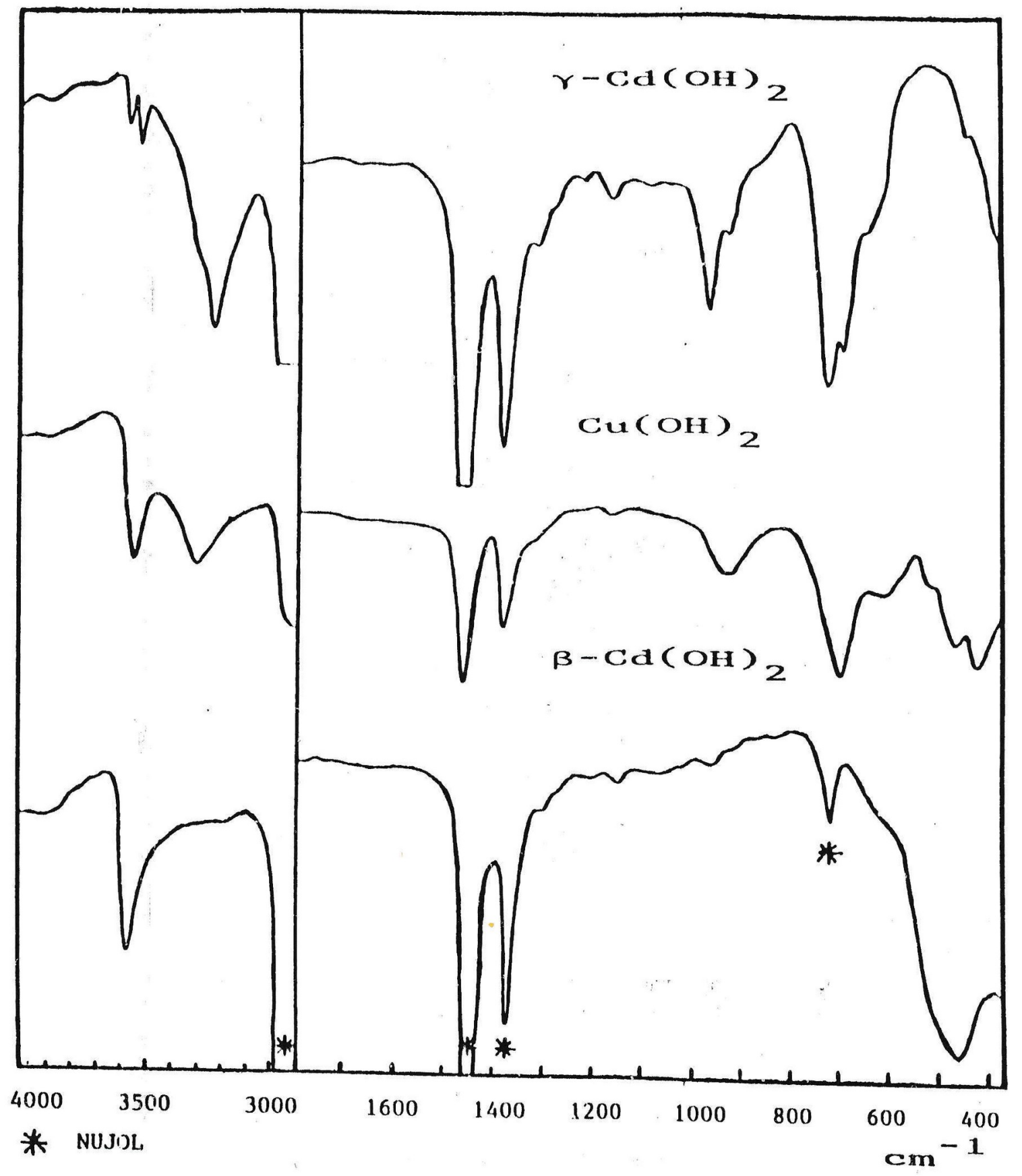

FIG. 2. SPECTRES INFRAROUGFS 